
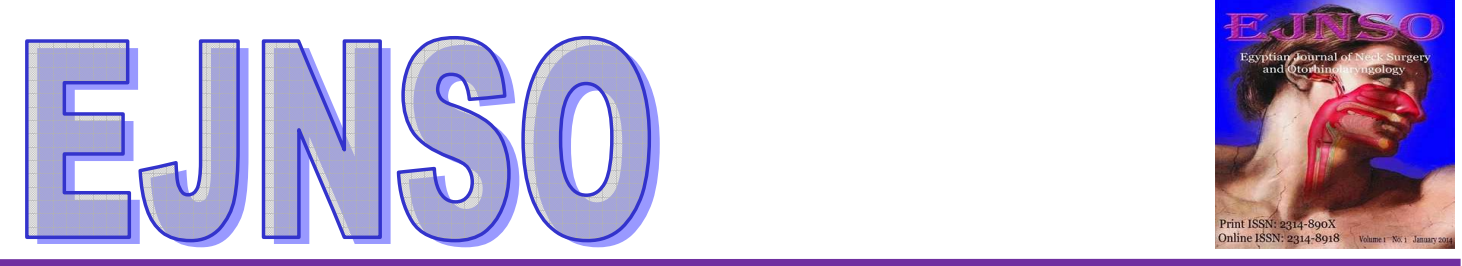

\title{
Does Tonsillectomy Have Impact on the Outcome of PFAPA Syndrome in Children?
}

\author{
Ahmed E. Ahmed ${ }^{a}$ and Essam A. Abo Al-magd \\ a- Lecturer of Pediatrics, Pediatrics Department, Qena Faculty of Medicine, South Valley University, Egypt. \\ b- Lecturer of Otorhinolaryngology, E.N.T. Department, Qena Faculty of Medicine, South Valley University, Egypt.
}

Received: $14 / 2 / 2014$

Revised: 5/3/2014

Accepted: 29/5/2014

Keywords:

PFAPA syndrome, tonsillectomy

\begin{abstract}
:
The medical syndrome clinically characterized by the association of periodic fever, aphthous stomatitis, pharyngitis, and cervical lymphadenopathy. Renamed it PFAPA syndrome to underline the distinctiveness of the clockwork periodicity of events and thus include it in the differential diagnosis of periodic fever syndromes in children.

Objective: To assess the effectiveness of tonsillectomy in the management of children with PFAPA syndrome.

Patients and Methods: A prospective study carried out in collaboration between Pediatrics and Otolaryngology Departments, Qena Faculty of Medicine, South Valley University, Egypt in the period between January (2010) to December (2013) after approval from Qena Faculty of Medicine ethics committee. Thirty-six patients with age range from 20-52 months evaluated for periodic fever were included in the study who attended Qena University Hospital, South Valley University. Patients underwent long-term pediatrics and otorhinolaryngological assessments. Their parents were asked to keep monthly diaries with reports of any subsequent episodes, symptoms, and related signs. Patients received traditional medical therapies, and 36 patients underwent tonsillectomy due to the lack of lasting recovery. The associations between postoperative outcomes and age at tonsillectomy and the differences in the patients' condition before and after tonsillectomy were statistically tested. In addition, the removed tonsillar tissue was analyzed molecularly to evaluate concomitant infections. Results: All of the surgical patients reported a symptomatic improvement, with complete clinical recovery in 35 cases $(97.22 \%)$, follow up were done to all patients at 3,6 and 12 months after tonsillectomy (36 patients,32cases and 32 cases respectively) show complete clinical recovery.

Conclusions: Tonsillectomy operation is effective and appropriate in the management of PFAPA in children who do not improve spontaneously or do not respond to traditional medical therapy.

Recommendations: Otolaryngologists should be trained to recognize PFAPA syndrome, for which management consists of a regular and prolonged second level pediatric and otolaryngological follow-up, with surgery only after the failure of traditional medical therapy.
\end{abstract}

\section{1-Introduction:}

The medical syndrome clinically characterized by the association of periodic fever, aphthous stomatitis, pharyngitis, and cervical lymphadenopathy was first described in 1987 by Marshall el $\mathrm{al}^{1}$ and was subsequently called fever, aphthous stomatitis, pharyngitis, and cervical lymphadenopathy (FAPA) syndrome by Feder and Bialecki in $1989 .^{2}$ In the same year, Marshall et $\mathrm{al}^{3}$ renamed it PFAPA 
syndrome to underline the distinctiveness of the clockwork periodicity of events and thus include it in the differential diagnosis of periodic fever syndromes in children.

The diagnostic criteria for typical PFAPA syndrome according to Marshall et al., ${ }^{3}$ include the following: (1) onset of disease before 5 years of age; (2) regularly recurring, abrupt episodes of fever lasting about 5 days with constitutional symptoms associated with both (a) aphthous stomatitis and/or pharyngitis (with or without cervical adenitis) without other signs of respiratory tract infection and (b) acute inflammatory markers (leukocytosis or elevated erythrocyte sedimentation rate); (3) completely asymptomatic interval period, normal growth parameters, benign long-term course, and no sequelae; ( 4 ) exclusion of cyclic neutropenia; ( 5 ) exclusion of other periodic fevers; and (6) exclusion of immunodeficiency, autoimmune diseases, or chronic infections. Although the exact etiology of PFAPA syndrome is still unknown, one tempting hypothesis suggests that the presence of infections and aberrant cytokine regulation are successive steps in a single etiopathogenic process, well described by neologism "kinection" put forth by Long. ${ }^{4}$

On the basis of the partial clinical overlap between PFAPA syndrome and other periodic fevers that are known to be hereditary (ie, hyper-IgD syndrome, familial Mediterra nean fever, cyclic neutropenia), some authors have investigated the possible role of genetic abnormalities such as the Mediterranean fever gene mutations responsible for immunological deregulation ${ }^{5.6}$,however, there is still no convincing evidence of their occurrence in children with PFAPA syndrome.

In terms of treatment, in addition to traditional medical therapy, surgery has been judged to be an effective therapeutic option in a few, mainly retrospective, studies because it is capable of removing the supposed antigen in lymphatic tissue that triggers the autoimmune reaction generated in tonssilar parenchyma ${ }^{7}$.

The management of PFAPA syndrome in children was initially entrusted to pediatricians alone but is now considered to need the cooperation of an adequately trained ear, nose, and throat (ENT) specialist In this way, patients would be referred to specific secondlevel pediatric settings to confirm the diagnosis and establish the most appropriate treatment on the basis of an integrated medical and surgical therapeutic protocol. The treatment of PFAPA syndrome is still a matter of debate. Administration of antibiotics, non steroidal anti-inflamatory, acyclovir, acetylsalicylic acid and colchicines has been shown to be ineffective, apart from the reduction of fever induced by anti-inflamatory agents. On the contrary, the use of oral steroids (predsone or prednisolone) causes a dramatic resolution of febrile episodes although it dose not prevent their recurrence ${ }^{1,} 5,7$. This research was designed to assess the effectiveness of tonsillectomy in the management of children with PFAPA syndrome.

\section{Subjects and Methods:}

A prospective study was carried out in collaboration between Pediatrics and Otolaryngology Departments, Qena Faculty of Medicine, South Valley University, Egypt; in the period between December 2010 to December 2013- after approval from Qena Faculty of Medicine ethics committee. Thirtysix patients fulfilled the traditional diagnostic criteria for PFAPA syndrome according to the diagnostic criteria of Marshall et al. ${ }^{3}$.

Demographic data After excluding other causes of periodic fever, PFAPA syndrome was diagnosed after at least 6 months of clinical observation and careful examinations of parent-completed monthly diaries providing detailed descriptions of each episode, symptom, and related sign, including periodic fever, aphthous stomatitis, pharyngitis, cervical lymphadenopathy, abdominal pain, arthralgia, headache, nausea or vomiting, diarrhea, and anorexia, as well as any treatments received. During acute exacerbations, on at least 1 occasion, all of the patients underwent assessments of erythrocyte sedimentation rate; hemochromogen, C-reactive protein, and antistreptolysin $\mathrm{O}$ antibody levels; and pharyngeal swab positivity for group A B-hemolytic streptococcus (GABHS).

The occurrence of episodes was evaluated for each patient by means of a preoperative and postoperative score index of occurrence (SIO): i.e., the mean ratio between the number of episodes and the time from their onset (preoperatively) or from tonsillectomy (postoperatively). In the case of pharyngeal swab positivity or in the presence of clinical signs of concomitant infections, episodes were excluded from SIO computation.

The patients underwent periodic pediatric and otolaryngological assessments after tonsillectomy, and their parents were asked to continue completing monthly diaries with reports of any subsequent episodes. All the patients and their parents were informed about the aim of the study and gave their written consent to the procedure and follow-up.

Statistical analysis: 
The significance of the association between postoperative outcome (postoperative SIO, and the duration of residual episodes) and age at tonsillectomy was tested using SPSS version 20 and GraphPad Prism 5. Values were recorded as mean and standard deviation. $\mathrm{P}$ values $<0.05$ were considered significant.

\section{Results:}

The male patients represent $47.2 \%$ of the study patients, $69.4 \%$ of patients from Qena Governorate and 30.6\% from Aswan Governorate with the mean age $35.42 \pm 1.47 \mathrm{SE}$ months.

The mean age of onset was 18 months with a mean duration of the PFAPA syndrome was 18 months with the mean frequency of the syndrome was 4.7 every week. As regard seasonality, no seasonality reported in $75 \%$ of cases while $16.7 \%$ of cases reported in summer. As regard change in frequency of episodes, no frequency change reported in $50 \%$ of the studied cases, gradual decrease in frequency reported in $13.89 \%$, gradual increase in frequency reported in $33.33 \%$ and relapse after long-term remission in $2.78 \%$ of the studied patients with PFAPA syndrome.

Fever, pharyngitis, cervical lymphadenopathy and aphthous stomatitis reported in $100 \%, 69.44 \%, 66.67 \%$ and $44.44 \%$ respectively of the studied patients with PFAPA syndrome. The classic cluster of PFAPA syndrome reported in $61.11 \%$ of the studied patients with PFAPA syndrome.

Additionally, $83.3 \%$ of cases primary diagnosed by Pediatrician and $16.7 \%$ by ENT Physician. As regard laboratory investigations the mean WBC was 10986.11 \pm 2854.78 SD and the mean ESR was $63.74 \pm 16.29 \mathrm{SD}$.

Tonsillectomy was done to all studied patients with PFAPA syndrome with effectiveness in $97.22 \%$ and relapse in 1 case only $2.78 \%$. 
Table 1:-Demographic data of the patients with

\begin{tabular}{|l|c|}
\hline \multicolumn{1}{|c|}{ Symptoms } & $\begin{array}{c}\text { Frequency, no of patients } \\
(\mathbf{\%})\end{array}$ \\
\hline Fever & $36(100)$ \\
\hline Pharyngitis & $25(69.44)$ \\
\hline Cervical lymphadenopathy & $24(66.67)$ \\
\hline Aphthous stomatitis & $16(44.44)$ \\
\hline $\begin{array}{l}\text { Classic cluster (fever, } \\
\text { pharyngitis, lymphadenopathy, } \\
\text { aphthous stomatitis) }\end{array}$ & $22(61.11)$ \\
\hline
\end{tabular}

Table 3:- Symptoms reported by parents of children with PFAPA syndrome

\begin{tabular}{|c|c|}
\hline No of patients & 36 \\
\hline Male, $\mathrm{n}(\%)$ & $17(47.2)$ \\
\hline Female, $\mathrm{n}(\%)$ & $19(52.8)$ \\
\hline Origin & $25(69.4)$ \\
\hline Qena, $\mathrm{n}(\%)$ & $11(30.6)$ \\
\hline Aswan, $\mathrm{n}(\%)$ & $20.52 \pm 1.47$ \\
\hline Age of onset, months (mean \pm SE) & \\
\hline
\end{tabular}

Table 4:-Diagnosing physicians of patients with PFAPA and laboratory investigations.

\begin{tabular}{|c|l|}
\hline & No of patients \\
\hline Diagnosing Physician & \\
\hline Primary Pediatrician, $\mathrm{n}(\%)$ & $30(83.3 \%)$ \\
\hline ENT, n (\%) & $6(16.7 \%)$ \\
\hline Time to diagnosis, monthes (range) & $35.42(20-52)$ \\
\hline Investigations & \\
\hline WBC, (Mean \pm SD) & $10986.11 \pm 2854.78$ \\
\hline ESR, (Mean \pm SD) & $63.74 \pm 16.29$ \\
\hline
\end{tabular}

\section{Discussion:}

According to the modified diagnostic criteria for Marshall's/PFAPA syndrome by Thomas et $\mathrm{al}^{7}$; the syndrome was diagnosed by the following: periodic fever in absence of upper respiratory tract infection with at least one of the other clinical signs (aphthous stomatitis, pharyngitis, adenilis) after exclusion of cyclic neutropenia and a completely asymptomatic interval between episodes with normal growth and development.

Although medical therapy is currently used in patients with PFAPA syndrome both preventively (with antibiotics therapy) and during acute exacerbations (with corticosteroids), there is little
PFAPA syndrome.

Table 2:-Clinical characteristics of PFAPA episodes.

\begin{tabular}{|c|c|}
\hline Onset of PFAPA, months (range) & $32(20-52)$ \\
\hline Duration of episode, months (range) & $18(10-28)$ \\
\hline Maximal temperature, ${ }^{\circ} \mathrm{C}$ (range) & $\begin{array}{c}40.1(38.5- \\
41.2)\end{array}$ \\
\hline Days of temperature, $38.3^{\circ} \mathrm{C}$ (range) & $4.5(1-14)$ \\
\hline Frequency, every week (range) & $4.7(4-5)$ \\
\hline \multicolumn{2}{|l|}{ Seasonality } \\
\hline No seasonality, n (\%) & $27(75)$ \\
\hline More in the winter, $\mathrm{n}(\%)$ & $3(8.3)$ \\
\hline More in the summer, $\mathrm{n}(\%)$ & $6(16.7)$ \\
\hline \multicolumn{2}{|l|}{ Frequency of episodes } \\
\hline Periodic fever, n (\%) & $36(100)$ \\
\hline Pharyngitis, $\mathrm{n}(\%)$ & $25(69.44)$ \\
\hline Aphthous stomatitis, n (\%) & $24(66.67)$ \\
\hline Adenitis, $\mathrm{n}(\%)$ & $16(44.44)$ \\
\hline \multicolumn{2}{|l|}{ Change in frequency of episodes } \\
\hline No frequency change, $\mathrm{n}(\%)$ & $18(50)$ \\
\hline Gradually decreased frequency, n (\%) & $5(13.89)$ \\
\hline Gradually increased frequency, n (\%) & $12(33.33)$ \\
\hline $\begin{array}{l}\text { Relapse after long-term remission, } \mathrm{n} \\
(\%)\end{array}$ & $1(2.78)$ \\
\hline
\end{tabular}

Table 5:-Treatment of PFAPA episodes.

\begin{tabular}{|c|l|}
\hline Treatment & $\begin{array}{l}\text { No of } \\
\text { patients }\end{array}$ \\
\hline Antibiotic, $\mathrm{n}(\%)$ & $36(100 \%)$ \\
\hline $\begin{array}{l}\text { Effectiveness of antibiotic treatment, } \mathrm{n} \\
(\%)\end{array}$ & $0(0.00 \%)$ \\
\hline Tonsillectomy, n (\%) & $36(100 \%)$ \\
\hline Time to tonsillectomy, months (range) & $53.89(33-72)$ \\
\hline $\begin{array}{l}\text { Recurrence of the disease after } \\
\text { tonsillectomy }\end{array}$ & $1(2.78 \%)$ \\
\hline
\end{tabular}

evidence that it has any notable impact on the natural history of the syndrome,(8) and it is not used in some countries such as Finland ${ }^{9}$. Furthermore, the effectiveness of tonsillectomy in patients with PFAPA syndrome is still a matter of debate, and only a few articles have been published $^{7,4}$, most of these support the efficacy of tonsillectomy, with complete recovery in $64 \%$ to $100 \%$ of cases $^{7,10,11,12,13}$, but the possibility of an incomplete recovery or a stationary clinical picture is well known $^{7,8,13,14,15}$. Long et al. ${ }^{4}$ have recently expressed some hesitancy about the effectiveness of tonsillectomy in children with PFAPA 
syndrome, based on the heterogeneity of the surgical success rate $e^{7,11}$, the tendency for spontaneous resolution of the syndrome, the limited series of patients reported, and the scarcity of prospective studies. In line with previously published findings ${ }^{11,12,13}$, our results document complete clinical recovery or a significant clinical improvement in $97.22 \%$ of the treated cases, and further occasional relapses after a transient clinical remission in $1(2.78 \%)$. Isolated aphthous stomatitis occasionally presented in 16 cases $(44.44 \%)$ but without any further PFAPA episodes;. for our knowledge, similar findings have not been previously reported, although Thomas et al. ${ }^{7}$ described persistent recurrent aphthous despite a reduction in febrile episode in 2 of 11 patients who underwent tonsillectomy however it is not clear whether this was associated with other PFAPA symptoms.

The patients with postoperative recurrences had less severe symptomatic clusters compared with before surgery; periodic lever and pharyngitis were the only symptoms in 1 patient and was accompanied by pharyngitis and headache. Published data indicate the possible role of microorganisms in PFAFA syndrome, as the chronic viral infections and periodic episodes of high fever, pharyngitis, and cervical adenitis suggest their direct or indirect involvement ${ }^{16,17}$.

Our results documented complete clinical recovery or a significant clinical improvement in $97.22 \%$ of the treated patients. These results were in line with previously published finding ${ }^{7,13,18}$. Only selected cases underwent tonsillectomy, i.e., the children with more severe clinical features unresponsive to traditional medical therapy who experienced increasingly frequent recurrences of PFAPA episodes over time in whom the frequency of the episodes did not change ${ }^{11}$.
Most of the previous studies were retrospective, and their postoperative follow-up periods were not always predetermined. They sometimes included children who actually had recurrent infections as children with PFAPA syndrome $^{8,}{ }^{19}$. To our knowledge, only 1 randomized controlled trial on PFAPA syndrome has been published ${ }^{12}$, although it presents some patients not fulfilling the accepted diagnostic criteria for PFAPA syndrome ${ }^{9,19}$. On the contrary, our case series only included patients strictly fulfilling the diagnostic criteria for PFAPA syndrome. Moreover during recurrences regular pharyngeal swabs for the assessment of GABHS positivity were useful to exclude acute infectious processes the occurrence of GABHS positivity, observed in no more than 1 event, was not considered a PFAPA episode and thus was not included in SIO computation.

In addition, we suspect that patients with occasional further episodes are sometimes considered completely recovered. For example, Renko et al. ${ }^{18}$ claimed that all 14 of their surgically treated patients showed a complete clinical recovery, although 4 experienced 1 episode compatible with periodic fever during the 6 months after tonsillectomy.

Finally, we believe that ENT specialists who evaluate recurrent upper airway infections in children should be trained to recognize this hardly debilitating syndrome to set up an appropriate therapeutic protocol with the cooperation of second-level pediatric facilities, deputed to the exclusion of other causes of periodic fever.

In conclusion, tonsillectomy operation is effective and appropriate in the management of PFAPA in children who do not improve spontaneously or do not respond to traditional medical therapy. 


\section{Recommendations:}

ENT specialists should be trained to recognize PFAPA syndrome, for which the current management consists of regular and prolonged clinical observation within an integrated otolaryngological and pediatric diagnostic and therapeutic protocol. However, randomized trials with more conspicuous case series could be useful to achieve clearer data, since the etiology is still unknown and currently adopted therapies are, at present, just alluring attempts.

\section{References:}

1. Marshall GS, Edwards KM, Butler J, Lawton AR. Syndrome of periodic fever, pharyngitis, and aphthous stomatitis. J Pediatr. 1987;110(1 ):43-46.

2. Feder HMJ Jr, Bialecki CA. Periodic fever associated with aphthous stomatitis, pharyngitis and cervical adenitis. Pediatr Infect Dis J. 1989;8(3):186-187.

3. Marshall GS, Edwards KM, Lawton AR. PFAPA syndrome. Pediatr Infect Dis J. 1989;8(9):658659.

4. Long SS. Syndrome of periodic fever, aphthous stomatitis, pharyngitis, and adenitis (PFAPA) what it isn't what is it? J Pediatr. 1999; 135(1): $1-5$.

5. Padeh S, Brezniak N, Zemer D, et al. Periodic fever, aphthous stomatitis, pharyngitis, and adenopathy syndrome: clinical characteristics and outcome. J Pediatr. 1999;135(1):98-101.

6. Cazeneuve C, Genevieve S, Amselem S, Hentgen V, Hau I, Reinert P. MEFV gene analysis in PFAPA. J Pediatr. 2003;143(1):140141.

7. Thomas KT, Feder HMJ Jr, Lawton AR, Edwards KM. Periodic fever syndrome in children. J Pediatr. 1999;135(1):15-21.

8. Renko M, Uhari M. The role of tonsillectomy in management of periodic lever, aphthous stomatitis, pharyngitis, and adenopathy: unanswered questions (reply) J Pediatr. 2008;152(5):743.

9. Hernandez-Bou S.GinerM, Plaza AM, Sierra Jl. Martin Mateos MA. PFAPA syndrome: with regard to a case. Allergol Immunopathol (Madr). 2003;31 (4):236-239.

10. Galanakis E, Papadakis CE, Giannoussi E, Karatzanis AD, Bitsori M, Helidonis ES. PFAPA syndrome in children evaluated for tonsillectomy. Arch Dis Child. 2002; 86(6):434435 .

11. Marque M, Guillot B. Bessis D. Thalidomide for treatment of PFAPA syndrome [letter]. Oral
Surg Oral Med Oral Pathol Oral Radiol Endod. 2007; 103(3) 306- 307.

12. Wong KK. Finlay JC, Moxham JP. Role of Tonsillectomy in PFAPA syndrome. Arch Otolaryngol Head Neck Surg. 2008;134(1):1619.

13. Abele-Horn M, Busch U, Nitschko $H$, et al. Molecular approaches to diagnosis of pulmonary diseases due to Mycoplasma pneumoniae. J Clin Microbiol. 1998; 36(2):548-551.

14. Parikh SR, Reiter ER, Kenna MA, Roberson D. Utility of tonsillectomy in 2 patients with the syndrome of periodic fever, aphthous stomatitis, pharyngitis, and cervical adenitis. Arch Otolaryngol Head Neck Surg. 2003;129(6) :670673.

15. Licameli G, Jeffrey J, Luz J, Jones D, Kenna M. Effect of Adenotonsillectomy in PFAPA Syndrome. Arch Otolaryngol Head Neck Surg. 2008;134(2):136-140.

16. Duppenthaler A. Recurrent febrile episodesnormal, periodic fever syndrome or immunodeficiency? TherUmsch. 2006;63(10):667-671.

17. Spalding SJ, Hashkes PJ. The role of tonsillectomy in management of periodic fever, aphthous stomatitis, pharyngitis, and adenopathy: unanswered questions [letter]. J Pediatr. 2008;152(5):742-743.

18. Renko M, Salo E, Putto-Laurila A, et al. A randomized, controlled trial of tonsillectomy in periodic fever, aphthous stomatitis, pharyngitis, and adenitis syndrome. J Pediatr. 2007;151 (3):289-292.

19. Ogose T. Tonsidectomy for periodic fever, aphthous stomatitis, pharyngitis, and adenitis syndrome is not always successful [letter]. J Pediatr. 2008;152(5):742.

20.Tanaka H, Westesson PL, Wilbur OC. Leiomyosarcoma of the maxillary sinus: CT and MRI findings. BrJ Radiol. 1998;71(842):221224.

21. Tastier D, Somekh E, Dalai I. PFAPA syndrome: new clinical aspects disclosed. Arch 